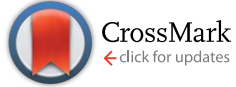

Cite this: J. Mater. Chem. A, 2017, 5, 1967

Received 19th December 2016 Accepted 10th January 2017

DOI: 10.1039/c6ta10886e

www.rsc.org/MaterialsA

\section{A facile strategy towards a highly accessible and hydrostable MOF-phase within hybrid polyHIPEs through in situ metal-oxide recrystallization $\uparrow$}

\author{
Matjaž Mazaj, ${ }^{* a}$ Nataša Zabukovec Logar, ${ }^{\text {ac }}$ Ema Žagar ${ }^{\text {b }}$ and Sebastijan Kovačič*b
}

HKUST-1(Cu) and MOF-5(Zn)apolyHIPE hybrid materials were prepared using a metal salt-free technique, wherein metal-organic frameworks were in situ generated from the CuO- and ZnO-nanoparticles through secondary recrystallization. The solid-to-MOF transformation has proven to be a feasible and effective technique for preparing MOF@ polyHIPE hybrid materials with a high MOF content of more than $75 \mathrm{wt} \%$. The MOF phase within the hybrid polyHIPEs as disclosed herein exhibits superior micropore accessibility, structure hydrostability and durable $\mathrm{CO}_{2}$ adsorption capacity under humid conditions, not achievable with any of the previously reported methods.

Porous metal-organic frameworks (MOFs), crystalline solids with a well-defined porous structure, are a rapidly emerging class of multifunctional materials, which can be applied in various fields of physical and chemical processing. ${ }^{1}$ Utilization of potentially useful MOFs is often hindered due to the low hydrostability or the challenges of shaping. ${ }^{2}$ To widen the practical utility of MOFs and enhance their durability, synthetic strategies that will deliver MOFs in a new, application-specific configurations are vital. Several bottom-up (using pre-formed templates) ${ }^{3}$ and top-down (using pre-synthesised MOFs) ${ }^{4}$ synthetic strategies of shaping have been already applied. A significant amount of effort was made in depositing MOFs nanocrystals onto porous substrates like ceramic, ${ }^{5}$ silica, ${ }^{6}$ alumina $^{7}$ or within the mixed-matrix membrane (MMM) systems. ${ }^{8}$ Embedding MOFs into the organic (polymeric) matrices is an appealing combination while hybrids maintain the shape and flexibility of the polymeric supports and exhibit

\footnotetext{
${ }^{a}$ National Institute of Chemistry, Department for Inorganic Chemistry and Technology, Hajdrihova 19, 1000 Ljubljana, Slovenia.E-mail: matjaz.mazaj@ki.si

${ }^{b}$ National Institute of Chemistry, Department for Polymer Chemistry and Technology, Hajdrihova 19, 1000 Ljubljana, Slovenia. E-mail: sebastijan.kovacic@ki.si

${ }^{c}$ University of Nova Gorica, Vipavska 13, 5000 Nova Gorica, Slovenia

$\dagger$ Electronic supplementary information (ESI) available: Experimental procedures, XRD, TGA, $\mathrm{N}_{2}$ sorption isotherms, and micropore accessibility calculations. See DOI: $10.1039 /$ c6ta10886e
}

the microporosity and high surface area of MOFs. Particularly interesting are microcellular polymeric foams as matrices, since their macropores serve as "highways" providing an unobstructed flow for hosting molecules that are aiming to access active-sites within the MOF's microporosity. Pore geometry, sizes and surface functionalities in porous polymeric matrices can easily be adjusted through one of the various methodologies. ${ }^{9}$ Among the methodologies available for preparing porous polymers, high internal phase emulsion (HIPE) templating and polyHIPEs thereof are especially intriguing. ${ }^{10}$ The major advantage of polyHIPEs is very high porosity (between 74 and 95 vol\%) and unique void and window structures that are highly 3D-interconnected with voids typically between 5 and $20 \mu \mathrm{m}$ in diameter. A number of hybrids based on various MOFs and polyHIPEs have been developed through different synthetic routes: conventional solvothermal synthesis of the MOFs within the pre-formed polyHIPEs, ${ }^{11,12}$ incorporation of pre-formed MOFs into the HIPEs following polymerization, ${ }^{13}$ and simultaneous formation of MOFs and polyHIPEs. ${ }^{14}$ However, the hybrids designed by these three approaches are all facing the same problems associated with the MOF phase (i) chemical and hydrolytic instability in monomeric solutions, (ii) low loadings and (iii) micropore clogging. Recently, an efficient synthetic strategy, the so-called seeded growth, has been introduced for the MOF synthesis, where nano- or microparticles were used as seeds that nucleate the MOFs. ${ }^{15}$ Seed deposition offers the ability to use particles (e.g. metal-oxides) to locate the MOF nucleation and growth in preferential areas.

Herein, we extend the concept of seeded growth strategy to the MOFs@polyHIPE hybrids for the first time, by using preformed metal-oxide@polyHIPE nanocomposites. Key for this endeavour is the use of metal-oxide nanoparticles $\left(\mathrm{MO}_{x}\right.$-NPs) embedded within the polymer walls as seeds and as metal ion reservoirs for MOF growth in the presence of appropriate organic ligands (i.e. BTC $=1,3,5$-benzenetricarboxylic acid or $\mathrm{BDC}=1,4$-benzenedicarboxylic acid). The synthetic approach as disclosed herein has several advantages over the existing ones regarding MOFs: (i) the micropore clogging by the 
emulsion components is negligible, yielding high sorption accessibility after polymerization; (ii) MOF stability in HIPEs is no longer an issue, since the direct contact with the monomeric solution is avoided. This allows very sensitive MOF systems to be incorporated into polyHIPE; (iii) MOF loading is conditioned by the content of $\mathrm{MO}_{x}$-NPs within the hybrid that can be very high (up to $40 \mathrm{wt} \%$ according to the monomers).

First, microcellular nanocomposite foams ( $\mu \mathrm{CNF}$ ) based on $\mathrm{ZnO}$ and $\mathrm{CuO}$ NPs were prepared according to the protocols recently published. ${ }^{16} \mathrm{~A}$ mixture of dicyclopentadiene (DCPD; 20 vol\%) as a monomer, oleic acid coated NPs (30 wt $\%$ of NPs according to the monomer, Table S1 $\dagger$ ), and surfactant Pluronic L-121 (7 vol\% according to monomer) as a stabilizer were used as the organic (continuous) phase, while the internal (droplet) phase was deionized water ( $80 \mathrm{vol} \%)$. This formulation was subsequently polymerized upon the addition of the ringopening metathesis polymerization (ROMP) initiator (M2, see Scheme 1). The porous structures of the purified and dried $\mu \mathrm{CNF}$ were characterized using scanning electron microscopy (SEM). The $\mu$ CNF exhibited the highly interconnected, opencell, porous structures typical of polyHIPEs, as seen in Fig. 1C and $\mathrm{D}$. The void and the interconnecting window diameters are around 7 and $2 \mu \mathrm{m}$, respectively. Afterwards, $\mathrm{Cu}-$ and $\mathrm{Zn}$-based MOFs were successfully recrystallized under the solvothermal conditions from the corresponding $\mu \mathrm{CNF}$ into the HKUST- $1(\mathrm{Cu})$ @polyHIPE and MOF-5(Zn)@polyHIPE hybrids. Scanning electron microscopy (SEM) revealed the fully preserved polyHIPE morphology with octahedral (HKUST-1) or cubic (MOF-5) shaped crystallites, embedded within the void walls (Fig. 1A and B). PolyHIPE's void and window sizes remain in the same range as they were before the recrystallization.

$\mathrm{MO}_{x}$-NPs are completely recrystallized into the MOF phase as evidenced by XRD (Fig. 2 and $\mathrm{S} 1 \dagger$ ), which reveal no visible diffraction peaks belonging to the corresponding $\mathrm{MO}_{x}$ in any case. However, broadening of the XRD peaks and increased

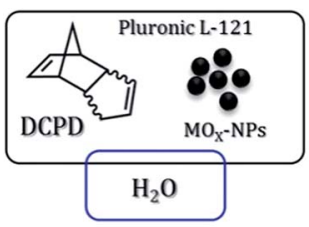

HIPE

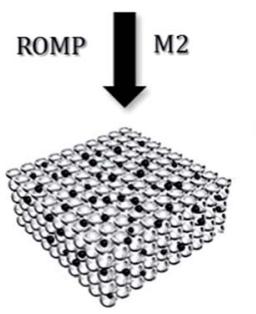

MOx@polyHIPE

Nanocomposites

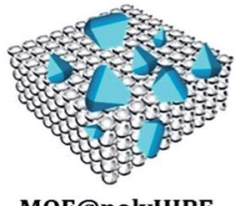

MOF@polyHIPE Hybrids

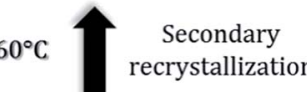

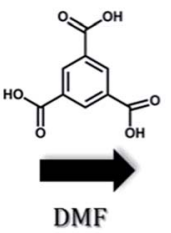

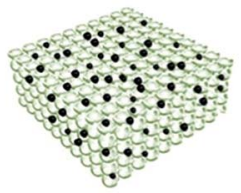

Scheme 1 Schematic representation of the MOF nanocrystals grown through the secondary recrystallization of the $M O_{x}-N P s$ within the polyHIPEs.
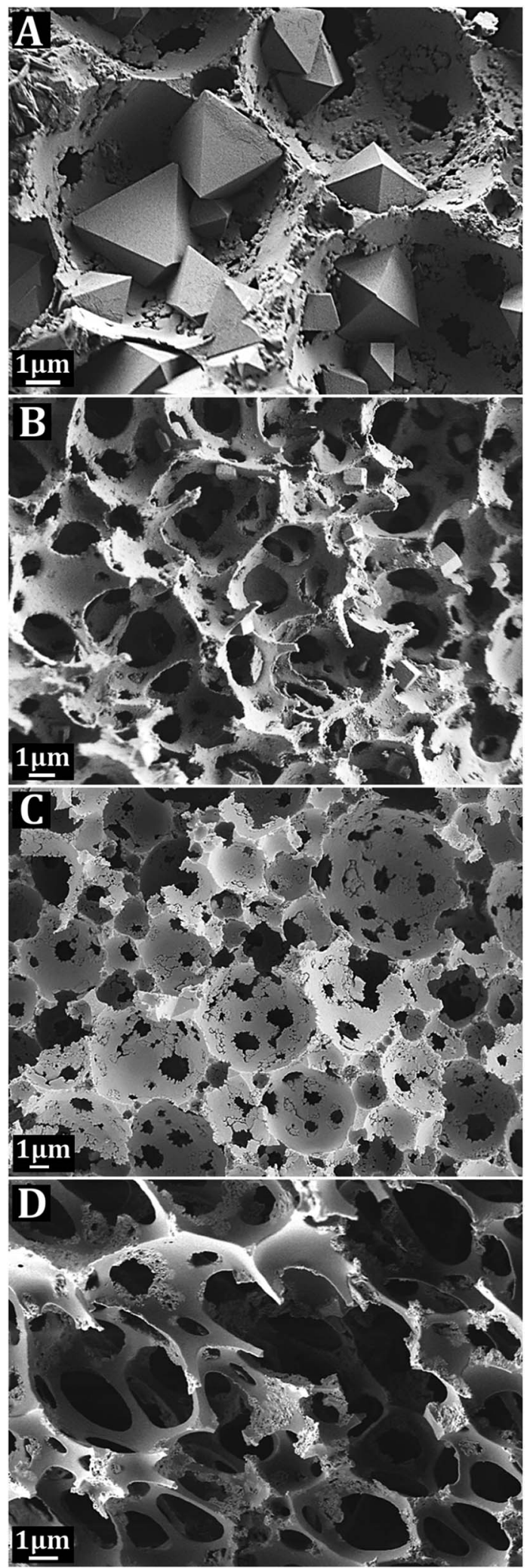

Fig. 1 Scanning electron micrographs of (A) HKUST-1(apolyHIPE (B) MOF-5apolyHIPE (C) CuO $\mu \mathrm{CNF}$ and (D) ZnO $\mu \mathrm{CNF}$.

background of the composite patterns can result in the overestimation of the MOF recrystallization rate due to the potential overlapping of the peaks corresponding to the metal oxides. 

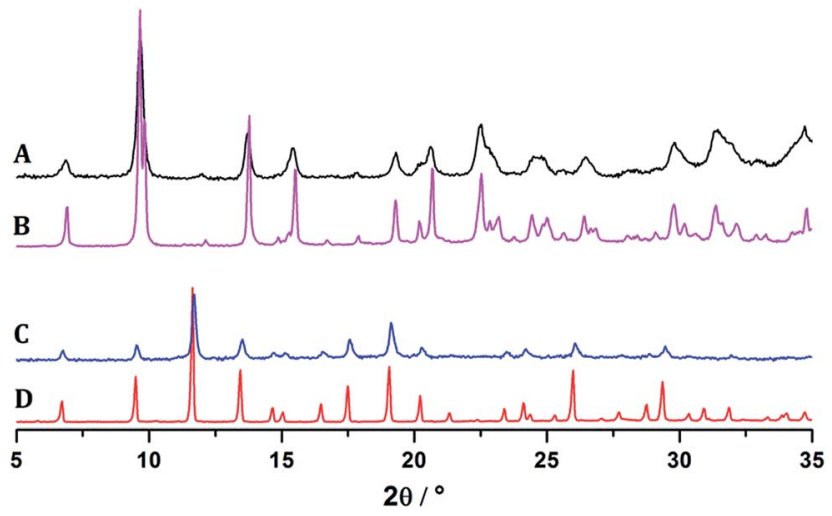

Fig. 2 XRD patterns of (A) MOF-5apolyHIPE (B) parent MOF-5 (C) HKUST-1@polyHIPE and (D) parent HKUST-1.

Therefore, TGA was performed to determine the MOF contents within the hybrids as well as to cross-check the recrystallization efficiency (Fig. S2 and S3†). Taking into account the MOF-5 formula, i.e. $\mathrm{Zn}_{4} \mathrm{O}(\mathrm{BDC})_{3}$, the solvent content, and the total weight loss of $\mathrm{ZnO} \mu \mathrm{CNF}$ (78.1 wt\%), the theoretical amount of MOF-5 after complete recrystallization should be $55.1 \mathrm{wt} \%$ (Fig. S2 and S3 $\uparrow$ ). The TGA of the MOF-5@polyHIPE hybrid material shows a weight loss of $24 \%$ due to the solvent removal and $57.8 \mathrm{wt} \%$ due to the decomposition of organic compounds. The calculated amount of the MOF-5 in the hybrid material perfectly matches the theoretical value of $55.1 \mathrm{wt} \%$. The measured amount of HKUST- 1 within the hybrid material was found to be 75.4 wt\% and thus almost attains the theoretical value extracted from the thermogram of the $\mathrm{CuO} \mu \mathrm{CNF}$ hybrid material (76.5 wt\%). Higher contribution of HKUST-1 within the polyHIPE matrix can be explained by higher CuO loading within the polyHIPE ( $28 \mathrm{wt} \%$ for $\mathrm{CuO}$ vs. $22 \mathrm{wt} \%$ for $\mathrm{ZnO}$ ). TGA confirmed almost complete transformation of the $\mathrm{MO}_{x}$-NPs into the MOF phase in both cases as it was already suggested by XRD measurements. Integration of MOFs within the polyHIPE matrix provides the opportunity to retain MOF's high porosity and accessibility for hosting molecules which is very important in applications such as catalysis or gas storage and separation. ${ }^{11}$ However, avoiding micropore blocking through the preparation of MOF@polyHIPE hybrid materials remains a big challenge. Secondary recrystallization as disclosed herein seems to be a promising solution. Pure HKUST-1 and MOF-5 powders show typical type I isotherm as determined by the BET method with the specific surface areas $\left(S_{\mathrm{BET}}\right)$ of 1211 and $2501 \mathrm{~m}^{2} \mathrm{~g}^{-1}$ for HKUST-1 and MOF-5, respectively. On the other hand, the pure $\mu$ CNFs have negligible surface areas with no accessible microporosity. After recrystallization of the $\mu \mathrm{CNFs}$ the $S_{\mathrm{BET}}$ of newly obtained hybrid materials increases significantly. In both cases, $S_{\mathrm{BET}}$ shows about half of the values as were measured for the parent MOF materials, i.e. 794 and $1298 \mathrm{~m}^{2} \mathrm{~g}^{-1}$ for the HKUST-1@polyHIPE and MOF5@polyHIPE, respectively. In both cases, the isotherms show a significant increase of the $\mathrm{N}_{2}$ uptake in the $p / p_{\mathrm{o}}$ region up to 0.1 , which can be assigned to the presence of the micropores. The MOF-5@polyHIPE hybrid material further exhibits a small hysteresis loop in the $p / p_{\text {o }}$ region between 0.85 and 1.0 most possibly due to the new mesopores created in the intercrystalline space of the MOF-5 or the interparticle space between MOF-5 crystals and the polyHIPE framework (Fig. 3). The drop of the surface area of hybrid materials goes mainly on the expense of the polyHIPE matrix presence, which does not contain any measurable microporosity. Moreover, secondary recrystallization is a post-polymerization process in which no emulsion components can clog the micropores. Therefore, it is sensible to suspect that the polymer matrix itself partially blocks the entry into the micropores. In order to estimate the quantity of the potentially blocked micropores the accessibility of MOF sorption sites, which is defined as a ratio between measured and expected BET surface areas, was calculated. The expected surface area was calculated taking into account the $S_{\mathrm{BET}}$ of the parent MOFs and their weight contribution within the hybrids (see ESI $\dagger$ ). The micropores' accessibility was found to the large extent unobstructed. HKUST-1@polyHIPE and MOF-5@polyHIPE hybrids exhibited $86 \%$ and $93 \%$ of the accessible micropores, respectively, which are among the highest values published for the MOF@polyHIPE hybrid materials (Table S2†). Slightly lower accessibility in the case of HKUST-1@polyHIPE can be related to the fact that the HKUST-1 crystals are more embedded within the polymer matrix as can be seen from the SEM micrographs (Fig. 1A and B).

MOF-5 and HKUST-1 crystals are known to exhibit high structure sensitivity towards moisture, which in large extent limits their applicability in various physical or chemical processes. Hydrostability was investigated for the synthesized hybrids in order to evaluate their functionalities under humid conditions. The hybrid materials and the parent MOFs were exposed to $98 \%$ of relative humidity for 3 days at room temperature. Both parent MOFs underwent the hydrolysis process already after $24 \mathrm{~h}$, which is manifested in the case of HKUST-1 by significant reduction of crystallinity and appearance of additional nonporous phase, whereas the structure of MOF-5 is completely recrystallized as can be seen from the

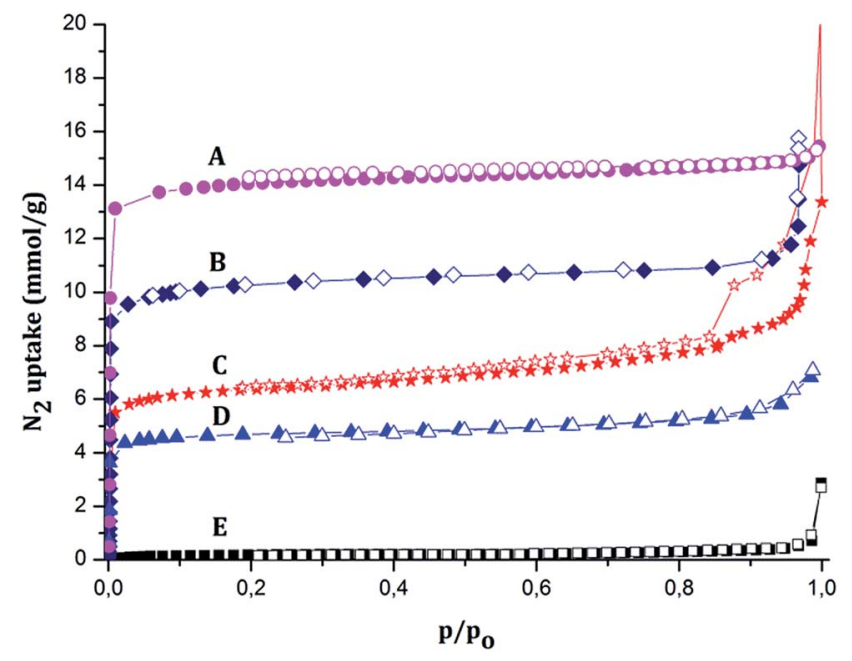

Fig. $3 \mathrm{~N}_{2}$ sorption isotherms measured at $77 \mathrm{~K}$ of (A) parent MOF-5, (B) parent HKUST-1, (C) MOF-5apolyHIPE hybrid, (D) HKUST-1@ polyHIPE hybrid, and (E) ZnO $\mu \mathrm{CNF}$. 
XRDs (Fig. S1 $\dagger$ ). ${ }^{17}$ On the other hand, the HKUST-1 and MOF-5 phases within the hybrid materials remained unchanged (Fig. S1†). A slight decrease of the reflection intensities and broadening of the peaks indicate partial amorphization of the MOF phase, however, the nonporous hydrous phase which commonly forms from the bulk MOF-5 did not occur. The $\mathrm{N}_{2}$ isotherms are consistent with the XRD results. The exposure of the HKUST-1@polyHIPE hybrid material in a humid environment did not have any significant negative effect on the porosity as indicated by only a slight decrease in the $S_{\mathrm{BET}}$ of the humidity treated sample (from 794 to $777 \mathrm{~m}^{2} \mathrm{~g}^{-1}$ ). The deterioration of the porosity is somewhat more pronounced in the case of MOF5@polyHIPE after humidity tests, wherein $S_{\mathrm{BET}}$ decreases for about 30\% (from 1298 to $910 \mathrm{~m}^{2} \mathrm{~g}^{-1}$ ) (Fig. S4†). The reason for such a profound improvement of the MOF hydrostability is due to the inherently hydrophobic nature of the poly(DCPD) polyHIPE matrix that repels the water vapour and protects the MOFs.

HKUST-1@polyHIPE and MOF-5@polyHIPE hybrid materials were further tested for the $\mathrm{CO}_{2}$ uptake under humid conditions. $\mathrm{CO}_{2}$ capture under the realistic conditions, e.g. $\mathrm{CO}_{2}$ removal from flue gases, includes also the presence of other components (most notably $5-7 \%$ water vapour). ${ }^{18}$ While the presence of water vapour significantly reduces the $\mathrm{CO}_{2}$ adsorption capacity of the promising porous materials, most of the $\mathrm{CO}_{2}$ adsorption tests are done under dry conditions. ${ }^{19}$ In our case, $\mathrm{CO}_{2}$ cycling experiments were performed under sequential $\mathrm{CO}_{2}$ adsorption at $1 \mathrm{bar}$ and $25{ }^{\circ} \mathrm{C}$ followed by exposure to $50 \%$ humidity. Both hybrid materials, i.e. HKUST-1@polyHIPE and MOF-5@polyHIPE (Fig. 4B and D), showed high durability. After 20 cycles, the $\mathrm{CO}_{2}$ sorption capacity of the HKUST-1@polyHIPE remained constant at around $108 \mathrm{mg} \mathrm{g}^{-1}$. In the case of MOF5@polyHIPE the capacity decreases for about 35\% (from 38 to $25 \mathrm{mg} \mathrm{g}^{-1}$ ), which is consistent with the loss of porosity as revealed by $\mathrm{N}_{2}$ sorption analysis. On the contrary, the parent HKUST-1 was continuously losing the ability to adsorb $\mathrm{CO}_{2}$ and

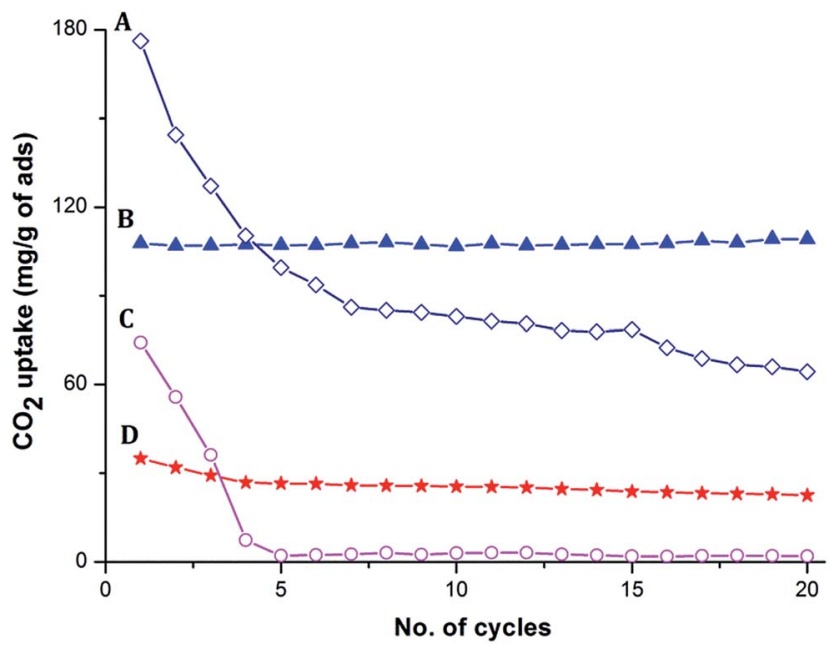

Fig. $4 \quad \mathrm{CO}_{2}$ adsorption cycling (A) parent HKUST-1, (B) HKUST1apolyHIPE hybrid, (C) parent MOF-5 and (D) MOF-5apolyHIPE hybrid. after 20 cycles exhibited only approximately $40 \%$ of the initial capacity. In the case of parent MOF-5, the decrease was even more pronounced and after 5 cycles the adsorption capacity became negligible, indicating its complete transformation into nonporous hydrous Zn-terephthalate.

\section{Conclusions}

A new synthetic route to prepare MOF@polyHIPE hybrid materials via secondary recrystallization of the pre-synthesized metal-oxide@polyHIPE nanocomposites is disclosed. This synthetic approach is a step forward in the MOF@polymer heterostructure design. It overcomes the issues of limited loadings, chemical instability and micropore clogging associated with the MOF phase once incorporated into the polymer framework. By avoiding a direct contact between the MOFs and the monomeric solution, we enable the incorporation of various sensible (unstable) MOFs into a polyHIPE structure. Referring to the advantages of the secondary recrystallization approach, the HKUST-1@polyHIPE and MOF-5@polyHIPE hybrid materials were synthesized and exhibit a high MOF phase loading with pronounced micropore accessibility. Both hybrid materials further show improved structure resistivity upon water vapour exposure, offering the functionality of the water-sensitive MOF hybrid materials under humid conditions. The structure-function relationship of the newly obtained hybrid material under humid conditions was further tested for $\mathrm{CO}_{2}$ uptake/exposure to $50 \%$ humidity cycling. After 20 cycles, the $\mathrm{CO}_{2}$ sorption capacity of the hybrid MOF@polyHIPE materials does not change significantly, showing high synergism of both components constituting the hybrid materials.

\section{Conflict of interest}

The authors declare no competing financial interest.

\section{Acknowledgements}

The authors gratefully acknowledge the financial support of the Ministry of Higher Education, Science and Technology of the Republic of Slovenia, and the Slovenian Research Agency (Program No. P2-0145 and P1-0021).

\section{Notes and references}

1 S. Kitagawa, R. Kitaura and S. Noro, Angew. Chem., Int. Ed., 2004, 43, 2334-2375; X. Zhang, W. Wang, Z. Hu, G. Wang and K. Uvdal, Coord. Chem. Rev., 2015, 284, 206-235; Y. Cui, B. Li, H. He, W. Zhou, B. Chen and G. Qian, Acc. Chem. Res., 2016, 49, 483-493.

2 V. Colombo, S. Galli, H. J. Choi, G. D. Han, A. Maspero, G. Palmisano, N. Masciocchi and J. R. Long, Chem. Sci., 2011, 2, 1311-1319; Q.-L. Zhu and Q. Xu, Chem. Soc. Rev., 2014, 43, 5468-5512.

3 S. Aguado, J. Canivet and D. Farrusseng, Chem. Commun., 2010, 46, 7999-8001; S. Sorribas, B. Zornoza, C. Tillez and 
J. Coronas, Chem. Commun., 2012, 48, 9388-9390; H. J. Lee, W. Cho and M. Oh, Chem. Commun., 2012, 48, 221-223.

4 H. B. Tanh Jeazet, C. Staudt and C. Janiak, Chem. Commun., 2012, 48, 2140-2142; T. Rodenas, I. Luz, G. Prieto, B. Seoane, H. Miro, A. Corma, F. Kapteijn, F. X. L. Llabrés Xamena and J. Gascon, Nat. Mater., 2014, 14, 48-55; A. Sabetghadam, B. Seoane, D. Keskin, N. Duim, T. Rodenas, S. Shahid, S. Sorribas, C. L. Guillouzer, G. Clet, C. Tellez, M. Daturi, J. Coronas, F. Kapteijn and J. Gascon, Adv. Funct. Mater., 2016, 26, 3154-3163.

5 T. Granato, F. Testa and R. Olivo, Microporous Mesoporous Mater., 2012, 153, 236-246.

6 A. Sachse, R. Ameloot, B. Coq, F. Fajula, B. Coasne, D. De Vos and A. Galarneau, Chem. Commun., 2012, 48, 4749-4751.

7 E. V. Ramos-Fernandez, M. Garcia-Domingos, J. JuanAlcañiz, J. Gascon and F. Kapteijn, Appl. Catal., A, 2011, 391, 261-267.

8 B. Seoane, J. Coronas, I. Gascon, M. E. Benavides, O. Karvan, J. Caro, F. Kapteijn and J. Gascon, Chem. Soc. Rev., 2015, 44, 2421-2454.

9 D. Wu, F. Xu, B. Sun, R. Fu, H. He and K. Matyjaszewski, Chem. Rev., 2012, 112, 3959-4015.

10 N. R. Cameron, P. Krajnc and M. S. Silverstein, Colloidal Templating, in Porous Polymers, ed. M. S. Silverstein, N. R. Cameron and M. A. Hillmyer, John Wiley \& Sons, Inc., Hoboken, NJ, USA, 2011.

11 M. G. Schwab, I. Senkovska, M. Rose, M. Koch, J. Pahnke, G. Jonschker and S. Kaskel, Adv. Eng. Mater., 2008, 10, 1151-1155.
12 L. D. O'Neill, H. Zhang and D. Bradshaw, J. Mater. Chem., 2010, 20, 5720-5726; M. L. Pinto, S. Dias and J. Pires, ACS Appl. Mater. Interfaces, 2013, 5, 2360-2363.

13 M. Wickenheisser and C. Janiak, Microporous Mesoporous Mater., 2015, 204, 242-250; S. Kovačič, M. Mazaj, M. Ješelnik, D. Pahovnik, E. Žagar, C. Slugovc and N. Zabukovec Logar, Macromol. Rapid Commun., 2015, 36, 1605-1611; M. Wickenheisser, T. Paul and C. Janiak, Microporous Mesoporous Mater., 2016, 220, 258; H. Zhu, Q. Zhang and S. Zhu, Chem.-Eur. J., 2016, 22, 8751-8755.

14 C. L. Calvez, M. Zouboulaki, C. Petit, L. Peeva and N. Shirshova, RSC Adv., 2016, 6, 17314-17317.

15 C. M. Doherty, D. Buso, A. J. Hill, S. Furukawa, S. Kitagawa and P. Falcaro, Acc. Chem. Res., 2014, 47, 396-405; G. Zhan and H. C. Zeng, Chem. Commun., 2017, 53, 72-81.

16 S. Kovačič, N. B. Matsko, G. Ferk and C. Slugovc, J. Mater. Chem. A, 2013, 1, 7971-7978; S. Kovačič, A. Anžlovar, B. Erjavec, G. Kapun, N. B. Matsko, M. Zigon, E. Zagar, A. Pintar and C. Slugovc, ACS Appl. Mater. Interfaces, 2014, 6, 19075-19081.

17 Y. Ming, J. Purewal, J. Yang, C. Xu, R. Soltis, J. Warner, M. Veenstra, M. Gaab, U. Müller and D. J. Siegel, Langmuir, 2015, 31, 4988-4995; M. Schlesinger, S. Schulze, M. Hietschold and M. Mehring, Microporous Mesoporous Mater., 2010, 132, 121-127.

18 D. M. D'Alessandro, B. Smit and J. R. Long, Angew. Chem., Int. Ed., 2010, 49, 6058-6082.

19 Y. Wang and M. D. LeVan, J. Chem. Eng. Data, 2010, 55, 31893195; J. Liu, A. I. Benin, A. M. B. Furtado, P. Jakubczak, R. R. Willis and M. D. LeVan, Langmuir, 2011, 27, 1145111456. 